\title{
Synergistic impact of pre-diabetes and immunosuppressants on the risk of diabetes mellitus during treatment of glomerulonephritis and renal vasculitis
}

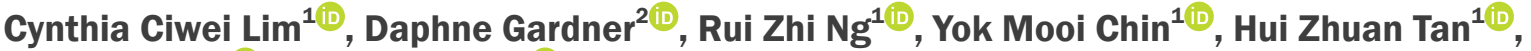 \\ Irene YJ Mok ${ }^{1(i)}$, Jason CJ Choo ${ }^{1(i)}$ \\ 'Department of Renal Medicine, Singapore General Hospital, Singapore \\ 2Department of Endocrinology, Singapore General Hospital, Singapore
}

Background: Glomerulonephritis is often treated with kidney-saving, but potentially diabetogenic immunosuppressants such as glucocorticosteroids and calcineurin inhibitors. Unfortunately, there are little data on dysglycemia before and after diagnosis and during treatment of glomerulonephritis. We aimed to evaluate the occurrence and risk factors for pre-diabetes and incident diabetes among non-diabetic patients with glomerular disease with or without treatment with immunosuppressants.

Methods: A single-center, retrospective cohort study was performed on 229 non-diabetic immunosuppressantnaïve adults diagnosed with glomerulonephritis and renal vasculitis. Patients with known diabetes and prior immunosuppressant treatment were excluded. Outcomes of new-onset pre-diabetes and new-onset diabetes were defined according to American Diabetic Association criteria.

Results: Pre-diabetes was present pre-biopsy in 74 of the 229 patients (32.3\%). During the median follow-up of 34.0 (23.3-47.5) months, 29 patients (12.7\%) developed new-onset diabetes and 58 (25.3\%) had new-onset prediabetes. Immunosuppressive therapy in patients with pre-existing pre-diabetes was associated with increased odds of new-onset diabetes compared to those without either risk factor (26.0\% versus 5.0\%; odds ratio, $6.67 ; 95 \%$ confidence interval [Cl], 1.41 to 31.64), $P=0.02$ ).

Conclusion: New-onset diabetes after immunosuppressant treatment occurred in one-quarter of patients with glomerulonephritis and pre-existing pre-diabetes. Physicians should screen for pre-diabetes when planning treatment with immunosuppressants, as its presence significantly increases the risk of diabetes mellitus.

Keywords: Follow-up studies, Glomerulonephritis, Hyperglycemia, Immunosuppression, Pre-diabetic state

Received February 6, 2020; Revised April 9, 2020;

Accepted April 26, 2020

Edited by Won Kim, Chonbuk National University, Jeonju, Republic of Korea

Correspondence: Cynthia Ciwei Lim

Department of Renal Medicine, Singapore General Hospital. Academia Level 3, College Road, Outram, Singapore 169856, Singapore. E-mail: cynthia.lim.c.w@singhealth.com.sg

Copyright () 2020 by The Korean Society of Nephrology

(a) This is an open-access article distributed under the terms of the Creative Commons Attribution Non-Commercial License (http://creativecommons. org/licenses/by-nc-nd/4.0/), which permits unrestricted non-commercial use, distribution, and reproduction in any medium, provided the original work is properly cited.

\section{Introduction}

Glomerulonephritis and renal vasculitis can lead to chronic kidney disease and account for $10 \%$ to $30 \%$ of incident end-stage renal diseases [1-3], costly illnesses that are associated with increased cardiovascular disease and mortality [4-6]. Although progression to kidney failure can be prevented by inducing and sustaining remission with immunosuppressive treatment [7], some commonly used immunosuppressants such as glucocorticosteroids and calcineurin inhibitors may have undesirable 
metabolic effects such as hyperglycemia. Diabetes and diabetes-related mortality are rapidly increasing globally, particularly in Asia [8,9], where Type 2 diabetes tends to occur at a younger age compared to Caucasians [10].

Although there are data demonstrating steroid-induced diabetes among patients with autoimmune conditions (in particular, inflammatory rheumatologic disease) [11], it is uncertain if this is applicable to those with glomerulonephritis. Patients with renal disease may have decreased glucose filtration, excretion, and inflammation-induced insulin resistance that predisposes them to hyperglycemia, while exhibiting reduced renal gluconeogenesis and insulin clearance, which lowers serum glucose as a result of impaired tubular function [12]. Moreover, immunosuppressant types and doses commonly used to treat glomerular disease vary considerably from other autoimmune conditions. For example, among the Systemic Lupus International Collaborating Clinics (SLICC) inception cohort of 1,700 patients, those with active renal disease were twice as likely to be prescribed glucocorticosteroids [13]. Use of concurrent immunosuppressants such as mycophenolate mofetil with lower prednisolone doses may reduce the adverse effects of high-dose steroid monotherapy, while other immunosuppressants such as calcineurin inhibitors are known to be diabetogenic [14].

The incidence and impact of dysglycemia is not welldescribed in patients being treated for glomerulonephritis. Therefore, there is a need to recognize risk factors that increase risk of diabetes among patients for whom clinicians may be planning treatment with potentially diabetogenic medications. Clinicians can then anticipate the risks of dysglycemia and consider personalizing immunosuppressant treatment for patients with glomerulonephritis. We aimed to assess the incidence, risk factors, and outcomes for new onset diabetes and pre-diabetes among patients with glomerular disease treated with immunosuppressant in usual clinical practice.

\section{Methods}

This was a retrospective cohort study of immunosuppressant-naïve, non-diabetic adults $\geq 21$ years of age with glomerulonephritis confirmed on kidney biopsy performed between January 2011 and July 2015 at Singapore General Hospital, an academic medical center and tertiary referral center. Subjects were identified from the kidney biopsy procedural log, which contains records of all native kidney biopsies performed. Exclusion criteria were treatment with immunosuppressive therapy before kidney biopsy and pre-existing diabetes mellitus (defined as previous physician diagnosis of diabetes, fasting glucose $\geq 126 \mathrm{mg} / \mathrm{dL}$, glycated hemoglobin (HbAlc) $\geq 6.5 \%$, or use of oral glucose lowering medications). Among the 343 immunosuppressive-naïve adults, we further excluded those without fasting glucose or HbAlc measurement within 6 months before the biopsy $(n=62)$ or during the follow-up $(n=52)$ since we could not ascertain their glycemic status.

Dysglycemia status was defined according to the American Diabetes Association criteria in 2010 for diabetes and pre-diabetes [15]. Pre-diabetes was defined as presence of impaired fasting glucose, i.e., fasting glucose between 100 and $125 \mathrm{mg} / \mathrm{dL}$, and/or HbAlc between 5.7\% and $6.4 \%$. New-onset diabetes was present if post-kidney biopsy fasting venous glucose was $\geq 126 \mathrm{mg} / \mathrm{dL}$ for two or more readings, HbAlc was $\geq 6.5 \%$, or if the patient required anti-diabetic medications during post-diagnosis clinical care and follow-up. Collectively, diabetes and pre-diabetes were termed dysglycemia. Data were retrieved until the last hospital visit or death.

Demographics, co-morbid diseases, and laboratory and medication data were retrieved from electronic medical records. Laboratory data collected included peak serum creatinine and urine protein-to-creatinine ratio within 1 month before the kidney biopsy; fasting triglycerides (TG), high-density lipoprotein cholesterol (HDL-C), and low-density lipoprotein cholesterol (LDL-C) within 24 months preceding biopsy; and $\mathrm{HbAlc}$ and fasting glucose within 6 months before immunosuppressant therapy. The TG-to-HDL-C ratio (TG/HDL-C) was calculated as it correlated with insulin resistance in diabetic and healthy cohorts [16-18]. Estimated glomerular filtration rate (eGFR) was calculated using the Chronic Kidney Disease Epidemiology Collaboration (CKD EPI) equation [19]. All laboratory investigations were conducted at our laboratory, which is accredited by the College of American Pathologists. Pharmacotherapy data retrieved included immunosuppressant medication prior to and after kidney biopsy, as well as peak daily dose of each immunosuppressant.

This study was conducted according to the Declaration of Helsinki and was approved by the Singhealth Central- 
ized Institutional Review Board (2015/2882) including waiver of informed consent for this medical records review.

Statistical analysis was performed using IBM SPSS Statistics 23.0 (IBM Corp., Armonk, NY, USA). Categorical variables were presented as proportions and continuous variables summarized as medians with interquartile ranges (IQR] (25th percentile, 75th percentile). The Pearson chi-square test was performed to compare categorical variables, while the Mann-Whitney $U$ test was used for non-normally distributed continuous variables. Stepwise binary logistic regression analysis was used to calculate the odds ratio (OR) and 95\% confidence interval (CI) for factors with a $P$-value $\leq 0.05$ associated with newonset diabetes on uni-variate analysis. All tests were twotailed, and statistical significance was defined as $P<0.05$.

\section{Results}

Table 1 shows the demographics, renal function, and metabolic parameters of the 229 non-diabetic patients with biopsy-proven glomerulonephritis not previously treated with immunosuppressants. The median age was
49.6 (IQR, 35.3-62.6) years. The median eGFR was 52.9 (26.2-90.6) $\mathrm{mL} / \mathrm{min} / 1.73 \mathrm{~m}^{2}$. More than half of the patients (58.1\%) had eGFR $<60 \mathrm{~mL} / \mathrm{min} / 1.73 \mathrm{~m}^{2}$, while twothirds of the adults $(n=150,65.5 \%)$ had nephrotic-range proteinuria.

Pre-diabetes was present in the biopsy in 74 of the 229 patients (32.3\%): 54 patients had fasting glucose between 100 and $125 \mathrm{mg} / \mathrm{dL}$, while 25 had HbAlc between 5.7\% and $6.4 \%$, and 13 satisfied both fasting glucose and $\mathrm{HbAlc}$ criteria for pre-diabetes. These patients tended to be older (53.7 [42.3-64.3] versus 47.4 [33.3-61.9] years, $P=0.04$ ), had higher systolic blood pressure (130 [120-139] versus 126 [114-140] mmHg, $P=0.03$ ), and exhibited worse renal function (eGFR $<60 \mathrm{~mL} / \mathrm{min} 1.73 \mathrm{~m}^{2}$ in $68.9 \%$ versus $52.9 \%, P=0.02)$ compared to those without pre-diabetes. The patients also had higher TG (2.0 [1.4-2.8] versus 1.6 [1.1-2.2] mmol/L, $P=0.008)$ and TG/HDL-C levels (1.5 [1.1-2.3] versus $1.2[0.7-1.9], P=0.004)$, possibly reflecting underlying insulin resistance $[16,18]$.

Table 2 shows the common glomerulonephritides in our cohort. Minimal change disease or focal segmental glomerulosclerosis was the most common diagnosis, followed by Immunoglobulin A nephropathy, membra-

Table 1. Comparison of clinical features in patients with glomerulonephritis according to immunosuppressive treatment

\begin{tabular}{|c|c|c|c|c|}
\hline & \multirow{2}{*}{$\begin{array}{l}\text { All patients } \\
(n=229)\end{array}$} & \multicolumn{2}{|c|}{ Immunosuppressive therapy after diagnosis } & \multirow{2}{*}{$P$ value } \\
\hline & & None $(n=64)$ & Treated $(n=165)$ & \\
\hline Age at diagnosis (yr) & $49.6(35.3-62.6)$ & $52.1(37.3-61.1)$ & $49.2(34.6-63.2)$ & 0.56 \\
\hline Male & $103(45.0)$ & $30(46.9)$ & $73(44.2)$ & 0.72 \\
\hline Hypertension & $133(58.1)$ & $48(75.0)$ & $85(51.5)$ & 0.001 \\
\hline Systolic BP (mmHg) & $130(118-140)$ & $130(110-146)$ & $130(120-139)$ & 0.57 \\
\hline Diastolic BP (mmHg) & $71(67-80)$ & $70(65-80)$ & $72(68-80)$ & 0.69 \\
\hline Dyslipidemia & $66(28.8)$ & $24(37.5)$ & $42(25.5)$ & 0.07 \\
\hline Serum creatinine (mg/dL) & $1.40(0.88-2.49)$ & $1.59(0.93-2.62)$ & $1.28(0.86-2.32)$ & 0.23 \\
\hline $\operatorname{eGFR}\left(\mathrm{mL} / \mathrm{min} / 1.73 \mathrm{~m}^{2}\right)$ & $52.9(26.2-90.6)$ & $48.7(25.4-87.8)$ & $53.9(27.3-91.7)$ & 0.34 \\
\hline CKD EPI eGFR < $60\left(\mathrm{~mL} / \mathrm{min} / 1.73 \mathrm{~m}^{2}\right)$ & $133(58.1)$ & $38(59.4)$ & $95(57.6)$ & 0.80 \\
\hline Urine protein-to-creatinine ratio (g/g) & $4.4(2.1-8.6)$ & $3.1(1.4-5.9)$ & $5.5(2.6-9.4)$ & $<0.001$ \\
\hline Fasting glucose (mg/dL) & & $94(88-100)$ & $92(86-101)$ & 0.44 \\
\hline HbA1c (\%) & $5.5(5.1-5.7)$ & $5.3(5.2-5.8)$ & $5.5(5.0-5.7)$ & 0.74 \\
\hline Pre-diabetes at baseline & $74(32.3)$ & $24(37.5)$ & $50(30.3)$ & 0.29 \\
\hline Triglyceride (mmol/L) & $1.8(1.1-2.4)$ & $1.6(1.0-2.4)$ & $1.8(1.2-2.4)$ & 0.35 \\
\hline LDL-C (mmol/L) & $3.9(2.8-6.3)$ & $3.5(2.6-5.0)$ & $4.2(2.9-6.7)$ & 0.03 \\
\hline $\mathrm{HDL}-\mathrm{C}$ (mmol/L) & $1.3(1.1-1.7)$ & $1.2(1.1-1.6)$ & $1.3(1.1-1.7)$ & 0.65 \\
\hline Triglyceride/HDL-C ratio & $1.3(0.8-1.9)$ & $1.2(0.7-2.0)$ & $1.4(0.8-1.9)$ & 0.54 \\
\hline
\end{tabular}

Categorical variables are expressed as number (\%) and compared using chi-square or Fisher's exact test as appropriate. Continuous variables are expressed as median (interquartile range) and compared using the Mann-Whitney $U$ test.

BP, blood pressure; CKD EPI, Chronic Kidney Disease Epidemiology Collaboration; eGFR, estimated glomerular filtration rate calculated using the CKD EPI formula; HbA1c, glycated hemoglobin; HDL-C, high-density lipoprotein cholesterol; LDL-C, low-density lipoprotein cholesterol. 
Table 2. Immunosuppressive treatment according to pathologic diagnoses ${ }^{a}$

\begin{tabular}{lcccc}
\hline & $\begin{array}{c}\text { Minimal change } \\
\text { disease or FSGS }\end{array}$ & $\begin{array}{c}\text { Immunoglobulin } \\
\text { A nephropathy }\end{array}$ & $\begin{array}{c}\text { Membranous } \\
\text { nephropathy }\end{array}$ & $\begin{array}{c}\text { Lupus } \\
\text { nephritis }\end{array}$ \\
\hline Number of patients & 67 & 57 & 33 & 31 \\
Proportion of cohort (\%) & 29.3 & 24.9 & & 14.4 \\
Immunosuppressant & & & & \\
$\quad$ Prednisolone & $45(67.2)$ & $33(57.9)$ & $23(69.7)$ & $29(93.5)$ \\
Methylprednisolone & $1(1.5)$ & $2(3.5)$ & $0(0.0)$ & $17(54.8)$ \\
Calcineurin inhibitor & $14(20.9)$ & $6(10.5)$ & $7(21.2)$ & $2(6.5)$ \\
Mycophenolate mofetil or sodium & $7(10.4)$ & $11(19.3)$ & $7(21.2)$ & $27(87.1)$ \\
Cyclophosphamide & $2(3.0)$ & $1(1.8)$ & $8(24.2)$ & $7(22.6)$ \\
\hline
\end{tabular}

Data are presented as number only or number (\%).

ANCA, antineutrophil cytoplasmic antibody; FSGS, focal segmental glomerulosclerosis.

${ }^{a}$ The four most frequent biopsy diagnoses among the 229 immunosuppressant-naïve non-diabetic adults are shown above, while etiologies including infectionassociated glomerulonephritis and ANCA-associated vasculitis constituted the remaining $17.9 \% .{ }^{\text {b}}$ The majority of patients with lupus nephritis also received hydroxychloroquine and only 1 received rituximab; none of the other 3 glomerulonephritide patients were treated with rituximab.

nous nephropathy, and lupus nephritis. Other etiologies including infection-associated glomerulonephritis and antineutrophil cytoplasmic antibody (ANCA)-associated vasculitis constituted the remaining $17.9 \%$ of diagnoses. Among the entire cohort of 229 immunosuppressantnaïve patients, immunosuppressive therapy was initiated after biopsy in 165 (72.1\%). Patients treated with immunosuppressants were more likely to have nephrotic-range proteinuria but less likely to be hypertensive compared to those who did not receive immunosuppressants (Table 1). Higher LDL-C levels among those patients treated with immunosuppressants may be due to greater proteinuria, as hypercholesterolemia is associated with nephrotic syndrome [20]. Age, TG/HDL ratio, and presence of prediabetes at baseline were not significantly different between the two groups.

Induction immunosuppressants were prednisolone ( $\mathrm{n}=$ $156,68.1 \%)$ with a peak daily dose of $50(30-60) \mathrm{mg}$, methylprednisolone $(\mathrm{n}=30,13.1 \%)$, calcineurin inhibitor ( $\mathrm{n}=35,15.3 \%)$, mycophenolate mofetil or sodium ( $\mathrm{n}=$ $58,25.3 \%)$, cyclophosphamide ( $\mathrm{n}=33,14.4 \%)$, and hydroxychloroquine ( $n=34,14.8 \%)$. Glucocorticosteroids, administered as monotherapy or more frequently in combination regimens according to international guidelines for each specific diagnosis [7], remained the backbone of immunosuppressive treatment among the most frequent glomerulonephritides in the cohort (Table 2). Choice of immunosuppressant did not significantly differ between patients with and without pre-diabetes at baseline. Methylprednisolone ( $17.6 \%$ versus $11.0 \%, P=0.16)$, predniso-

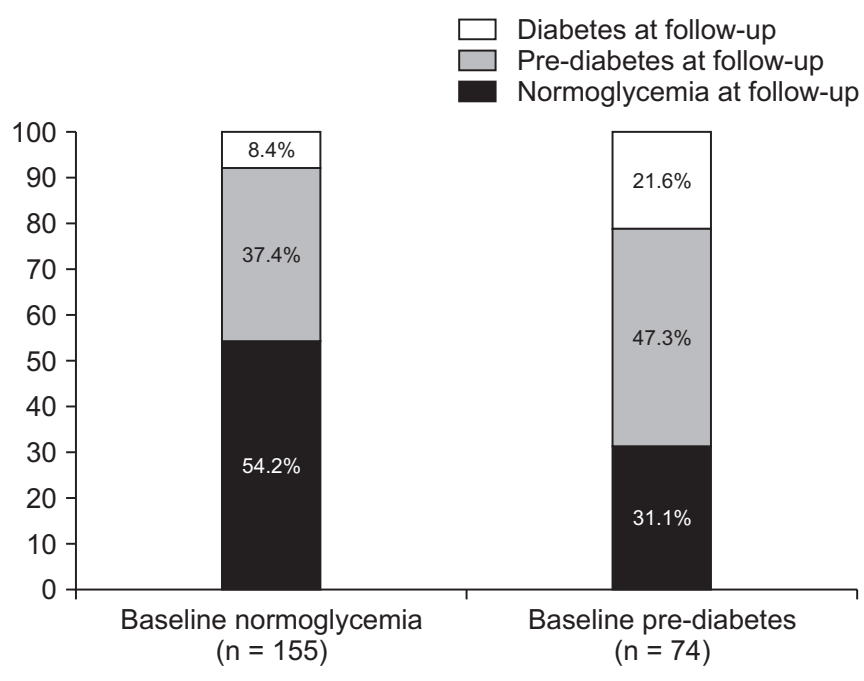

Figure 1. Glycemic outcomes during follow-up and treatment of glomerulonephritis, categorized according to pre-biopsy (baseline) glycemic status.

lone (63.5\% versus $70.3 \%, P=0.30)$, and peak daily prednisolone dose (50 [40-60] versus 50 [30-60] $\mathrm{mg}, P=0.42$ ) were similar between the groups, but there was a tendency for less frequent use of calcineurin inhibitors $(9.5 \%$ versus $18.1 \%, P=0.09$ ) among patients with pre-diabetes.

During the subsequent treatment and median followup of 34.0 (23.3-47.5) months, half the cohort ( $\mathrm{n}=122$, $53.3 \%)$ exhibited dysglycemia with either pre-diabetes or diabetes: 58 (25.3\%) had new-onset pre-diabetes, 35 (15.3\%) had persistent pre-diabetes, and 29 (12.7\%) had new-onset diabetes.

Among those who were normoglycemic at baseline, 
$58(37.4 \%)$ developed pre-diabetes, while $13(8.4 \%)$ had new-onset diabetes. Sixteen $(21.6 \%)$ of those with baseline pre-diabetes developed new-onset diabetes during treatment and follow-up (Fig. 1). Therefore, the presence of pre-diabetes at baseline increased the odds of developing diabetes during follow-up (OR, 3.01; 95\% CI, 1.36 to $6.65 ; P=0.006$ ).

Table 3 compares patients with and without new-onset diabetes and pre-diabetes. Those with new-onset diabetes were older, had higher baseline fasting glucose level and TG/HDL-C ratio, and had a higher number of treatments with methylprednisolone. Proteinuria tended to be more frequent among those with new-onset diabetes, although the difference was not statistically significant. There were no significant differences in use or peak daily doses of other immunosuppressants commonly used in this cohort (prednisolone, mycophenolate, and calcineurin inhibitors) compared with the occurrence of newonset diabetes. Multi-variable analysis adjusting for age, proteinuria, TG/HDL-C ratio, pre-diabetes status, and methylprednisolone use confirmed that pre-diabetes (adjusted OR, 2.63; 95\% CI, 1.06 to $6.52 ; P=0.04$ ) and methylprednisolone (adjusted OR, 4.14; 95\% CI, 1.40 to 12.26; $P=0.01$ ) were independently associated with newonset diabetes.

As part of personalized medicine to profile each individual's risk to aid physicians in planning and counselling for treatment, we further characterized the risk of new-onset diabetes among patients with glomerulonephritis by pre-diabetes status and use of immunosuppressants. Compared to those without baseline prediabetes and no immunosuppressant treatment, the

Table 3. Comparison of clinical characteristics between patients with and without new-onset diabetes and new-onset pre-diabetes

\begin{tabular}{|c|c|c|c|c|c|c|}
\hline & \multicolumn{2}{|c|}{ New-onset diabetes ${ }^{a}$} & \multirow{2}{*}{$P$ value } & \multicolumn{2}{|c|}{ New-onset pre-diabetes ${ }^{b}$} & \multirow{2}{*}{$P$ value } \\
\hline & No $(n=200)$ & Yes $(n=29)$ & & No $(n=84)^{c}$ & Yes $(n=58)$ & \\
\hline Age (yr) & $48.6(34.7-61.8)$ & $57.6(43.5-67.0)$ & 0.03 & $46.4(33.4-58.4)$ & $49.1(33.2-65.0)$ & 0.37 \\
\hline Male & $89(44.5)$ & $14(48.3)$ & 0.70 & 31 (36.9) & $26(44.8)$ & 0.34 \\
\hline Hypertension & $116(58.0)$ & $17(58.6)$ & 0.95 & $48(57.1)$ & $29(50.0)$ & 0.40 \\
\hline Systolic BP (mmHg) & $129(116-140)$ & $134(120-140)$ & 0.09 & $125(114-140)$ & $124(110-140)$ & 0.74 \\
\hline Diastolic BP (mmHg) & $70(67-80)$ & $74(68-82)$ & 0.42 & $71(62-80)$ & $70(63-80)$ & 0.19 \\
\hline Dyslipidemia & $54(27.0)$ & $12(41.1)$ & 0.11 & $22(26.2)$ & $16(27.6)$ & 0.85 \\
\hline Serum creatinine (mg/dL) & $1.30(0.85-2.50)$ & $1.57(1.11-2.50)$ & 0.18 & $1.11(0.75-1.96)$ & $1.18(0.84-1.97)$ & 0.92 \\
\hline eGFR $<60\left(\mathrm{~mL} / \mathrm{min} / 1.73 \mathrm{~m}^{2}\right)$ & $112(56.0)$ & $21(72.4)$ & 0.09 & $42(50.0)$ & $32(55.2)$ & 0.54 \\
\hline Urine protein-to-creatinine ratio (g/g) & $4.3(2.0-8.4)$ & $6.9(3.0-10.2)$ & 0.05 & $4.2(2.0-8.2)$ & $5.7(2.1-9.3)$ & 0.32 \\
\hline Fasting glucose at baseline (mg/dL) & $92(85-99)$ & $99(92-104)$ & 0.008 & $86(81-92)$ & $90(85-95)$ & 0.09 \\
\hline HbA1c at baseline (\%) & $5.4(5.1-5.7)$ & $5.6(5.0-5.9)$ & 0.23 & $5.2(4.9-5.4)$ & $5.2(4.9-5.5)$ & 0.56 \\
\hline Pre-diabetes at baseline & $58(29.0)$ & $16(55.2)$ & 0.005 & - & - & - \\
\hline Triglyceride (mmol/L) & $1.7(1.1-2.4)$ & $1.9(1.3-2.4)$ & 0.23 & $1.6(0.9-2.2)$ & $1.7(1.1-2.5)$ & 0.43 \\
\hline LDL-C (mmol/L) & $3.9(2.8-6.4)$ & $4.4(2.8-5.9)$ & 0.69 & $3.8(2.6-5.8)$ & $4.2(3.4-4.8)$ & 0.18 \\
\hline HDL-C (mmol/L) & $1.3(1.1-1.8)$ & $1.2(1.0-1.4)$ & 0.06 & $1.4(1.1-1.8)$ & $1.3(1.1-1.9)$ & 0.61 \\
\hline Triglyceride/HDL-C ratio & $1.3(0.7-1.9)$ & $1.6(1.1-2.4)$ & 0.03 & $1.0(0.6-1.7)$ & $1.2(0.7-1.9)$ & 0.32 \\
\hline Prednisolone & $133(66.5)$ & $23(79.3)$ & 0.16 & $56(66.7)$ & $43(74.1)$ & 0.34 \\
\hline Prednisolone peak daily dose (mg per day) & $50(30-60)$ & $40(30-60)$ & 0.38 & $42(30-60)$ & $50(30-60)$ & 0.46 \\
\hline Methylprednisolone & $21(10.5)$ & $9(31.0)$ & 0.006 & $7(8.3)$ & $6(10.3)$ & 0.68 \\
\hline Calcineurin inhibitor & $29(14.5)$ & $6(20.7)$ & 0.41 & $17(20.2)$ & $8(13.8)$ & 0.32 \\
\hline Mycophenolate mofetil or sodium & $50(25.0)$ & $8(27.6)$ & 0.76 & $21(25.0)$ & $20(34.5)$ & 0.22 \\
\hline
\end{tabular}

Categorical variables are expressed as number (\%) and compared using chi-square or Fisher's exact test as appropriate. Continuous variables are expressed as median (interquartile range) and compared using the Mann-Whitney $U$ test.

BP, blood pressure; CKD EPI, Chronic Kidney Disease Epidemiology Collaboration; eGFR, estimated glomerular filtration rate calculated using the CKD EPI formula; HbA1c, glycated hemoglobin; HDL-C, high-density lipoprotein cholesterol; LDL-C, low-density lipoprotein cholesterol.

${ }^{a}$ Diabetes was present when fasting venous glucose $\geq 126 \mathrm{mg} / \mathrm{dL}$ for 2 or more readings, HbA1c was $\geq 6.5 \%$, or if the patient required anti-diabetic medications during follow-up. ${ }^{b}$ Analysis for new-onset pre-diabetes after excluding patients with baseline pre-diabetes and those who developed new-onset diabetes. ${ }^{\mathrm{C} P a t i e n t s}$ who did not develop new onset-prediabetes were normoglycemic at baseline and remained normoglycemic during treatment and follow-up. 
Lim, et al. Dysglycemia in glomerulonephritis

Table 4. Incidence of diabetes according to baseline pre-diabetes and immunosuppressant treatment

\begin{tabular}{|c|c|c|c|c|c|}
\hline $\begin{array}{c}\text { Baseline } \\
\text { pre-diabetes }\end{array}$ & $\begin{array}{c}\text { Immunosuppressant } \\
\text { treatment }\end{array}$ & $\begin{array}{l}\text { Number of } \\
\text { patients }\end{array}$ & $\begin{array}{c}\text { New-onset diabetes } \\
\text { mellitus, } n(\%)\end{array}$ & $\begin{array}{c}\text { Odds ratio (95\% } \\
\text { confidence interval) }\end{array}$ & $P$ value \\
\hline No & No & 40 & $2(5.0)$ & 1.00 (reference) & - \\
\hline No & Yes & 115 & $11(9.6)$ & $2.01(0.42-9.48)$ & 0.38 \\
\hline Yes & No & 24 & $3(12.5)$ & $2.71(0.42-17.55)$ & 0.29 \\
\hline Yes & Yes & 50 & $13(26.0)$ & 6.67 (1.41-31.64) & 0.02 \\
\hline
\end{tabular}

Odds of new-onset diabetes among patients characterized by their baseline pre-diabetes status and whether they received immunosuppressant treatment, compared with the reference group which did not have baseline pre-diabetes and did not receive immunosuppressants.

risk of new-onset diabetes was highest in the group with baseline pre-diabetes and immunosuppressive treatment (Table 4). This high-risk group's median age was 54.7 (IQR, 42.7-65.0) years, with half being male (50\%) and the majority with nephrotic range proteinuria $(76.0 \%)$ and impaired renal function (eGFR $<60 \mathrm{~mL} / \mathrm{min} / 1.73$ $\mathrm{m}^{2}$ in $66 \%$ ). Prednisolone (94.0\%), methylprednisolone (26.0\%), cyclophosphamide (28.0\%), and mycophenolate mofetil or sodium $(26.0 \%)$ were the most frequently used immunosuppressants. Among the 13 patients who had new-onset diabetes, 8 required oral anti-diabetic medications and 1 required insulin therapy at the median 2.23 (0.25-9.87) months after the immunosuppressants were started.

\section{Discussion}

This study found that dysglycemia was present in half of the non-diabetic patients during follow-up and treatment for biopsy-proven glomerulonephritis. In particular, newonset diabetes occurred in one-quarter of those with baseline pre-diabetes and treated with immunosuppressants. Since prednisolone was the most frequently used immunosuppressant among the patients in this study, the diabetogenic effect of immunosuppressant therapy illustrated in this study may be largely attributed to steroid-induced diabetes. Steroids induce insulin resistance by reducing glucose uptake and use in cells (including glycogen synthesis) and enhancing hepatic gluconeogenesis [21]. Such effects likely exacerbated hyperglycemia in patients with pre-diabetes, whose baseline glucose level is higher than normal but does not meet the criteria for diabetes and are well-recognized to be at higher risk for future development of diabetes [15]. In comparison, the incidence of diabetes mellitus was $12.8 \%$ among 4,101 low-risk, local general population adult Malays and Indians followed-up over 6 years [22], while cohorts with autoimmune diseases treated with high-dose glucocorticosteroid mono-therapy reported more frequent occurrence $(20 \%$ to $40 \%)$ of steroid-induced diabetes $[23,24]$. In particular, this study identified that potent glucocorticosteroids such as methylprednisolone, typically administered as intravenous boluses of $500 \mathrm{mg}$ daily for $\mathbf{3}$ days in our institution, was associated with new-onset diabetes.

Despite elevated risks of diabetes and departmental recommendations to monitor fasting glucose at least two months after immunosuppressant therapy, we found patients without fasting glucose and HbAlc values within 6 months prior to immunosuppressant initiation ( $\mathrm{n}=27$, $16.4 \%$ of 165 ) and post-immunosuppressant treatment ( $\mathrm{n}=26 / 165,15.8 \%$ ). Fasting glucose was first measured $5.1(2.1-10.5)$ weeks after starting immunosuppressant treatment and subsequently much later at $11.0(4.7-20.3)$ weeks. This may reflect reduced awareness or conviction among physicians to risk-stratify and monitor for dysglycemia in the absence of consensus or evidencebased guidelines for evaluating glycemic parameters in glomerular diseases $[7,25]$. In contrast, the international guidelines for kidney transplant suggest that transplant recipients should be monitored with fasting glucose level at least weekly for 4 weeks and then every 3 months for 1 year [26].

The limitations of this study include its retrospective nature without data on markers of insulin resistance such as body mass index, waist-to-hip ratio, and fasting insulin level. Instead, we adjusted for TG/HDL-C ratio as a surrogate for insulin resistance [16,17]. Although most prior studies on steroid-induced diabetes based their diagnostic criteria on fasting blood glucose [11], there is concern that the latter may underestimate steroidinduced hyperglycemia, particularly when intermediateacting glucocorticosteroids such as oral prednisolone are administered as single morning doses [11]. We did not evaluate the risk of impaired glucose tolerance sepa- 
rately, but it was included within those with baseline prediabetes since it is our usual clinical practice to perform an oral glucose tolerarance test only if any individual exhibited impaired fasting glycemia. The low event rate may limit the power to detect significant associations for individual immunosuppressants such as calcineurin inhibitors, especially when their use was infrequent. It also limited the ability to adjust for multiple variables simultaneously in a logistic regression. Causality cannot be confirmed in this observational study, and the results are only hypothesis-generating. Despite these limitations, this study highlighted the considerable risk of new-onset diabetes for patients with glomerulonephritis and impaired fasting glycemia who receive immunosuppressive therapy. In addition, there may be a lack of awareness of metabolic complications and diabetic risk factors in clinicians caring for those with glomerulonephritis.

In summary, new-onset diabetes after immunosuppressant therapy occurred in one-quarter of our patients with glomerulonephritis and pre-existing pre-diabetes. Physicians should consider screening for dysglycemia during treatment and follow-up of the patients with glomerulonephritis, especially if they have pre-diabetes and were treated with immunosuppressants.

\section{Conflicts of interest}

Jason CJ Choo has served on Advisory Boards for Novartis and Pfizer with donation of honorarium to Singapore General Hospital to support research and education. The other authors have no conflicts of interest to declare.

\section{Acknowledgments}

The authors thank their colleagues in the Department of Renal Medicine for their support of this project.

\section{Authors' contributions}

Cynthia Ciwei Lim, Daphne Gardner, and Jason CJ Choo conceptualised the study and study design. Yok Mooi Chin and Cynthia Ciwei Lim collected data. Cynthia Ciwei Lim performed statistical analysis. Cynthia Ciwei Lim, Jason CJ Choo, and Daphne Gardner interpreted data. Cynthia Ciwei Lim, Rui Zhi Ng, and Hui Zhuan Tan prepared the manuscript. All authors provided critical in- tellectual input to the work and reviewed the final manuscript.

\section{References}

[1] Lim CC, Tan HZ, Hao Y, et al. Long-term renal outcomes in multi-ethnic Southeast Asians with lupus nephritis: a retrospective cohort study. Intern Med J 2018;48:1117-1123.

[2] Haynes R, Staplin N, Emberson J, et al.; SHARP Collaborative Group. Evaluating the contribution of the cause of kidney disease to prognosis in CKD: results from the Study of Heart and Renal Protection (SHARP). Am J Kidney Dis 2014;64:40-48.

[3] Wakai K, Nakai S, Kikuchi K, et al. Trends in incidence of end-stage renal disease in Japan, 1983-2000: age-adjusted and age-specific rates by gender and cause. Nephrol Dial Transplant 2004;19:2044-2052.

[4] Salonen T, Reina T, Oksa H, Sintonen H, Pasternack A. Cost analysis of renal replacement therapies in Finland. Am J Kidney Dis 2003;42:1228-1238.

[5] Lim CC, Teo BW, Ong PG, et al. Chronic kidney disease, cardiovascular disease and mortality: a prospective cohort study in a multi-ethnic Asian population. Eur J Prev Cardiol 2015;22:1018-1026.

[6] McDonald SP, Russ GR, Kerr PG, Collins JF; Australia and New Zealand Dialysis and Transplant Registry. ESRD in Australia and New Zealand at the end of the millennium: a report from the ANZDATA registry. Am J Kidney Dis 2002; 40:1122-1131.

[7] Kidney Disease: Improving Global Outcomes (KDIGO) Transplant Work Group. KDIGO clinical practice guideline for glomerulonephritis. Kidney Int 2012;2 Suppl 2:139-274.

[8] Danaei G, Finucane MM, Lu Y, et al.; Global Burden of Metabolic Risk Factors of Chronic Diseases Collaborating Group (Blood Glucose). National, regional, and global trends in fasting plasma glucose and diabetes prevalence since 1980: systematic analysis of health examination surveys and epidemiological studies with 370 country-years and 2.7 million participants. Lancet 2011;378:31-40.

[9] Global Burden of Metabolic Risk Factors for Chronic Diseases Collaboration. Cardiovascular disease, chronic kidney disease, and diabetes mortality burden of cardiometabolic risk factors from 1980 to 2010: a comparative risk assessment. Lancet Diabetes Endocrinol 2014;2:634-647.

[10] Kong AP, Xu G, Brown N, So WY, Ma RC, Chan JC. Diabetes and its comorbidities--where East meets West. Nat Rev En- 
docrinol 2013;9:537-547.

[11] Liu XX, Zhu XM, Miao Q, Ye HY, Zhang ZY, Li YM. Hyperglycemia induced by glucocorticoids in nondiabetic patients: a meta-analysis. Ann Nutr Metab 2014;65:324-332.

[12] Pecoits-Filho R, Abensur H, Betônico CC, et al. Interactions between kidney disease and diabetes: dangerous liaisons. Diabetol Metab Syndr 2016;8:50.

[13] Little J, Parker B, Lunt M, et al. Glucocorticoid use and factors associated with variability in this use in the Systemic Lupus International Collaborating Clinics inception cohort. Rheumatology (Oxford) 2018;57:677-687.

[14] Montori VM, Basu A, Erwin PJ, Velosa JA, Gabriel SE, Kudva YC. Posttransplantation diabetes: a systematic review of the literature. Diabetes Care 2002;25:583-592.

[15] American Diabetes Association. Diagnosis and classification of diabetes mellitus. Diabetes Care 2010;33 Suppl 1: S62-S69.

[16] Ren X, Chen ZA, Zheng S, et al. Association between triglyceride to HDL-C ratio (TG/HDL-C) and insulin resistance in Chinese patients with newly diagnosed type 2 diabetes mellitus. PLoS One 2016;11:e0154345.

[17] Kim-Dorner SJ, Deuster PA, Zeno SA, Remaley AT, Poth M. Should triglycerides and the triglycerides to high-density lipoprotein cholesterol ratio be used as surrogates for insulin resistance? Metabolism 2010;59:299-304.

[18] Sung KC, Park HY, Kim MJ, Reaven G. Metabolic markers associated with insulin resistance predict type 2 diabetes in Koreans with normal blood pressure or prehypertension. Cardiovasc Diabetol 2016;15:47.

[19] Levey AS, Stevens LA, Schmid CH, et al.; CKD-EPI (Chronic
Kidney Disease Epidemiology Collaboration). A new equation to estimate glomerular filtration rate. Ann Intern Med 2009;150:604-612.

[20] Haas ME, Levenson AE, Sun X, et al. The role of proprotein convertase subtilisin/kexin type 9 in nephrotic syndromeassociated hypercholesterolemia. Circulation 2016;134:6172.

[21] Kwon S, Hermayer KL, Hermayer K. Glucocorticoid-induced hyperglycemia. Am J Med Sci 2013;345:274-277.

[22] Chan JCY, Chee ML, Tan NYQ, Cheng CY, Wong TY, Sabanayagam C. Differential effect of body mass index on the incidence of diabetes and diabetic retinopathy in two Asian populations. Nutr Diabetes 2018;8:16.

[23] Uzu T, Harada T, Sakaguchi M, et al. Glucocorticoidinduced diabetes mellitus: prevalence and risk factors in primary renal diseases. Nephron Clin Pract 2007;105:c54c57.

[24] Miyawaki Y, Katsuyama T, Sada KE, et al. A retrospective observational study of glucocorticoid-induced diabetes mellitus with IgA nephropathy treated with tonsillectomy plus methylprednisolone pulse therapy. PLoS One 2017;12: e0178018.

[25] Beck L, Bomback AS, Choi MJ, et al. KDOQI US commentary on the 2012 KDIGO clinical practice guideline for glomerulonephritis. Am J Kidney Dis 2013;62:403-441.

[26] Kidney Disease: Improving Global Outcomes (KDIGO) Transplant Work Group. KDIGO clinical practice guideline for the care of kidney transplant recipients. Am J Transplant 2009;9 Suppl 3:S1-S155. 